\author{
KSENIA KUZMINYKH \\ Pädagogische Hochschule Ludwigsburg \\ ksenia.kuzminykh@ph-ludwigsburg.de
}

\title{
Erzählen im integrativen Deutschunterricht
}

\author{
Storytelling in literary and language teaching \\ of German
}

\begin{abstract}
The article starts with a discussion of the essential theories of storytelling, reading and writing, and focuses on the concept of reading-to-write. It discusses the use and the abuse of classical schema of storytelling - "the mouse". In the last step, using fragments of different children's books, the article develops an integrative literary and language teaching model for school lessons of German in a way which stimulates a highly functional receptive and productive handling of language semantics and performance.
\end{abstract}

KEYWORDS: storytelling, reading, writing, scaffolding, theories of storytelling, integrative literary and language teaching in school lessons of German.

SCHLÜSSELWORTE: Erzählen, Lesen, Schreiben, Thorien des Erzählens, integrativer Deutschunterricht für den Erwerb von literalen und sprachlichen Fähigkeiten.

\section{THEORIE DES ERZÄHLENS UND IHRE BEDEUTUNG FÜR DIE SCHULE}

Erzählen wurde in Abhängigkeit vom zu Grunde liegenden Konzept im Laufe der Zeit unterschiedlich definiert. Ehlich versteht darunter eine komplexe Form sprachlicher Handlung, die eine "Tiefenfunktion" aufweist (1980: 20). Diese besteht in der Herstellung einer gemeinsamen, den Sprachrezipienten inkludierenden Welt, einer Erzählwelt (Finnemann 2006), im Akt der Narration, und leitet sich vom Bedürfnis des Sprachproduzenten dem Sprachrezipienten, ein Ereignis zu erzählen, es in einer gemeinsamen Welt 
$\mathrm{zu}$ reinszenieren und dadurch Erfahrungen $\mathrm{zu}$ transferieren, her (Ehlich 1984). Rehbein fasst das Erzählen als eine "homileische Kommunikation" auf (Rehbein 1984: 71), die sich im Austausch und Freude an interessanten Erfahrungen manifestiert. Klassischer Erzähltextanalyse folgend, ist eine Erzählung eine komplementäre Einheit aus Story und Erzähldiskurs (Wenzel 2004). Labov und/and Walezky definieren Erzählen als eine verbale Technik der Erfahrungsrekapitulation, der referentielle und evaluative Funktionen immanent sind, und der ein bestimmtes Performanzmuster eine Normalform - zu Grunde liegt. Die zunächst genannte Funktion fokussiert die chronologische Reihenfolge der Erzählereignisse. Die evaluative erlaubt dem Erzähler, Akzentuierungen vorzunehmen (Labov \& Walezky 1973: 80). Für die Normalform sind folgende Phasen konstituierend: „Orientierung, Komplikation, Resultat, Evaluation und Coda" (Labov \& Walezky 1973: 124), die weitgehend mit den Phasen - Einleitung, in der Personen, Orte sowie Zeit des Geschehens konkretisiert werden, Höhepunkt oder der „Punkt maximaler Komplikation“ (Labov \& Walezky 1973: 116), Auflösung und Schluss - der klassischen, traditionellen Erzählung übereinstimmen. Dittmar und/and Thielicke heben einen Orientierungsteil, eine Komplikation, eine Evaluation und ein Resultat als Konstituenten hervor (Dittmar \& Thielicke 1979). Rumelhart definiert in der Story-Grammar-Theorie, Erzählungen als Texte, die von Ereignisfolgen mit einem klaren Ablauf und konsistenter Handlungsführung in meist chronologischer Reihenfolge bestimmt sind oder sich darauf zurückführen lassen (Rumelhart 1975). Grundlegend für seine Vorstellung vom Erzählen sind das Vorhandensein für die Darstellung relevanter Ergebnisse, ihre Linearisierung zu kohärenten Ereignisfolgen, die Etablierung eines die Erzählwürdigkeit begründenden Bruchs der $\mathrm{zu}$ erwartenden Ereignisverläufe und die emotionale Qualifizierung der dadurch zustande gekommenen Ereignisfolgen. Ähnlich modellieren das Erzählen Thorndyke (1977), Mandler und Johnson (1978), van Dijk (1980a) und van Dijk (1980c). Aufgegriffen und unter erzählerwerbstheoretischer Perspektive präzisiert und erweitert wird dieses Modell von der Forschergruppe um Boueke (Boueke, Schülein, Büscher, Terhorst \& Wolf 1995). In diesem Modell werden als Strukturierungsmarker Orientierung, Geschichte und Schluss pointiert. Jeder dieser Konstituenten gliedert sich in weitere Unterkonstituenten, die bestimmte Merkmale tragen, wie beispielsweise eine den emotionalen Bezug des Erzählers zum Erzählten verdeutlichende "Affektmarkierung“ (Boueke et al. 1995: 107) oder „Ereignisstrukturmarkierung", die dazu dient, besondere Ereignisse der Erzählung zu betonen (Boueke et al. 1995: 77) oder die Markierung der Diskontinuität (Boueke et al. 1995: 89). Für sein Erwerbsmodell der Erzählfähigkeit differenziert die Forschergruppe vier Typen des Erzählens - isoliert-enumerativ, linear- 
sequentiell, kontrastiv-diskontinuierlich und narrativ bzw. evaluativ-involvierend, dabei wird der zuletzt genannte Typ als der anzustrebende festgelegt. Den Schülerinnen und Schülern, die evaluativ-involvierend erzählen, wird demensprechend ein höheres Entwicklungsniveau zugeschrieben (dazu kritisch Wolf 2000; Becker 2001; Augst, Disselhoff, Henrich \& Pohl 2007; Augst 2010; Knapp 2001; Rank 1995). Quasthoff hebt als das konstituierende Merkmal einer Erzählung das Vorhandensein eines Planbruchs hervor, einer Bruchstelle aus der Sicht des einen Aktanten, die die Gesamtrealisierung des Plans und damit das Erreichen des Planziels zunichtemacht oder vorübergehend in Frage stellt (Quasthoff 1980). Becker kombiniert die narratologischen Grundlagen, indem sie bei der Konzeptualisierung erzähltheoretischer Basis folgende Elemente als notwendig bezeichnet: für das schriftliche Erzählen sind es neben Setting bzw. Exposition, Komplikation und Abschluss bzw. Auflösung, das Vorhandensein eines logischen Bruchs bzw. eines Planbruchs in dem antizipierten Ereignis und die Minimalbedingung der Ungewöhnlichkeit, die durch affektive und emotionale Komponente intensiviert wird. Diese anders formuliert, ist das Besondere von dem erzählt wird (Becker 2001: 41; Quasthoff 1980). Auch Fix spricht von einem „erzählwürdigen Ereignis, das - vermeintlich real - in der Vergangenheit sattgefunden hat" (Fix 2006: 94) und van Dijk von einem Interessantheitskriterium (van Dijk 1980a). In kritischer Auseinandersetzung zu Boueke et al. (1995) entwickeln Augst et al. (2007) ein ontogenetisches Strukturmodell - das Paradigma-Modell - zum schriftlichen Erzählen von fiktionalen Geschichten mit fünf Elementen "Setting-Planbruch-Spannung-Pointe-Coda" (Augst et al. 2007: 32). Als Stufen für die Erzählontogenese ausgehend von den in seinen Longitudinalstudien gewonnen Ergebnissen bestimmt er pränarrativ-rudimentäre, narrativ-konventionelle und literar-ästhetisch-individuelle (Augst 2010: 93). Eine besondere Stellung erhält in diesem Modell die Pointe als der "strukturelle narrative Kristallisationspunkt" und der Erzählton (Augst 2010: 64).

Bei allen skizzierten Theorien, ohne ihre Leistung in Frage zu stellen und zu minimieren, handelt es sich um extrahierte und abstrahierte Minimalkriterien, um eine a priori angenommene globale (Ur-)Struktur, ein Ideal der Erzählung, ein Performanztyp. Dazu gehören zeitliche Abfolge, das Besondere, die Pointe bzw. der Spannungsaufbau, der Planbruch und die Abgeschlossenheit der Handlung. Diese Vorstellung hat Vorzüge, doch entkleidet sie sich der kulturellen und historischen Spezifika und berücksichtigt die Wirklichkeit des Erzählens nicht ausreichend. Im schulischen Kontext werden die Vorstellungen von der prototypischen Erzählung, orientiert am abstrakten Kompetenzideal, erneut (didaktisch) reduziert und in Form einer „Mauskurvendramaturgie“ (Frederking, Krommer \& Maiwald 2012: 260), 
die zum „Inbegriff des Erzählens erhoben wurde“ (Maiwald 2011: 62), dargestellt und vermittelt. Das Performanzmuster fußt auf einer langen Tradition der narratio von Aristoteles, es wird partiell durch die Erzähltheorien gestützt, es erfüllt das Kriterium der Transparenz, lässt sich leicht erklären und visuell in Lehrwerken und Unterrichtshilfen darstellen (Kuzminykh 2009). Es suggeriert Einfachheit und hat einen hohen Wiedererkennungswert. Es lässt sich leicht in den von Schülerinnen und Schülern verfassten Erzählungen auch mittels Kriterienlisten überprüfen und entsprechend benoten, und korreliert weitgehend mit einer Vorstellung von einem wohlgeformten Schriftstück (Bildungsstandards im Fach Deutsch 2012), das mit einer bestimmten "Schreibstrategie" erstellbar ist (Fix 2008: 93), von einem Textideal. Das Performanzphänomen führt allerdings auch zur sprachlichen Stereotypisierung, erzeugt Klischees und Banalitäten. So stellt Maiwald fest, dass es in typischen Schüleraufsätzen auf dem Weg zum Höhepunkt gerne heißt "Gesagt, getan! oder Doch da! Am Höhepunkt selbst gibt es bevorzugt rasende Herzen, schlotternde Knie oder feuchte Hände" (Mailwald 2011: 62). Ähnliches diagnostiziert Karg (2009). Augst kommt zum Schluss, dass der klassische schulische Dreischritt besonders im Hinblick auf die emotionale Involviertheit nicht hinreichend ist. Er plädiert für das Vervollkommnen der literalen Fähigkeit des Erzählens über die Grenzen der Primär und Sekundarstufe I hinaus und zwar vor dem Hintergrund der Arbeit mit und an literarischen Texten (Augst 2010: 93). Problematisch ist die den einzelnen Phasen zugewiesene Funktion. Beispielsweise dient die Phase der Etablierung, in der Rekonstruktion von Raum und Zeit, von Orten und Personen erfolgt - also Setting, in der Terminologie von Story-Grammar-Theorie - der Sicherung des Verständnisses beim Rezipienten und führt beim Weglassen zu erheblichen Verständnisschwierigkeiten (Rehbein 1980: 77). Es ist zwar richtig, dass eine Etablierung bzw. Einleitung für das Verstehen (eventuell der Makro- und der Superstruktur) sowie für die Aktivierung von narrativen Schemata hilfreich sein kann (van Dijk 1980a), aber eine Voraussetzung für das Verstehen der Erzählung und für das Disambiguieren von Situationsmodellen ist eine Einleitung bzw. Etablierung nicht. Besonders vor dem Hintergrund des kognitionslinguistischen Forschungsfeldes (Kintsch 1998; Reusser \& Reusser-Weyeneth 1994; Schnotz 2006). Die Sinnzumessung entfaltet sich beim Durchgang durch den Text, indem Schritt für Schritt das Vorwissen und die Textimpulse zusammenspielen. Der Einstieg medias in res oder ultimas in res kann vorübergehend den Leser mit Irritationen herausfordern, da er mehrere Informationen und, ohnehin, mehrere Situationsmodelle aufgrund der Polyvalenz des Erzähltextes im Gedächtnis aktiviert halten soll, um inferences vornehmen, ausgesparte Beziehungen und Unbestimmtheitsstellen (Iser 1976) schließen und Kohärenz herstellen zu können. 
Allerdings können sich die Schwierigkeiten in diesen mentalen Schritten auch bei einem Text mit einer Einleitung einstellen. Ferner kann die Einleitung dysfunktional sein und irritieren, wenn beispielsweise beim Aktivieren eines bestimmten narrativen Schemas keine Einleitung, wie in Kurzgeschichten, erwartet wird.

Schülerinnen und Schüler erzählen oft ausgehend von ihren (vor-) und (außer)schulischen literarischen Erzählerfahrungen, vom nicht-diskursiven Wissen (Reckwitz 2012) und etablierten Vorstellungen vom Erzählen und Texten, die sie für Erzählungen halten. Was dies für den integrativen Unterricht und für den Erwerb von literalen und sprachlichen Fähigkeiten bedeutet wird im folgenden Kapitel thematisiert.

\section{SCHRIFTLICHES ERZÄHLEN: TRANSFORMATIONSPARADIGMATA}

Das schriftliche Erzählen vor dem Hintergrund literarischer Erfahrungen und Arbeit mit und an Erzählungen der Wirklichkeit (dem Erzähldiskurs) ist voraussetzungsreich: Der erste Schritt impliziert das Lesen und die Konstruktion eines Situationsmodells des Gelesenen. Dabei können Verstehen des Textes und das Erkennen der durch die literaturästhetische Geformtheit vermittelten Wirkung von Sprache und ihrer Funktionalität sowie eine besondere textuelle Verfasstheit ein unerhebliches Maß an kompensatorischer Arbeit verlangen. Die Leserintentionen bedingen wesentlich den Lese- und Verstehensvorgang. Im Sinne der von Rehbein (1984: 71) vorgeschlagenen "homileischen Kommunikation“ bedarf es ganz anderer Tiefe der Wissensbestände, als wenn die gelesenen Erzählungen eine Grundlage für das Verfassen von eigenen Texten bieten sollen. Es bedarf auch einer Fähigkeit, dieses Wissen zu aktivieren und heranzuziehen (Kuzminykh 2009). Das Gelesene und seine Verarbeitung werden zum Ausgangspunkt des eigenen Schreib-

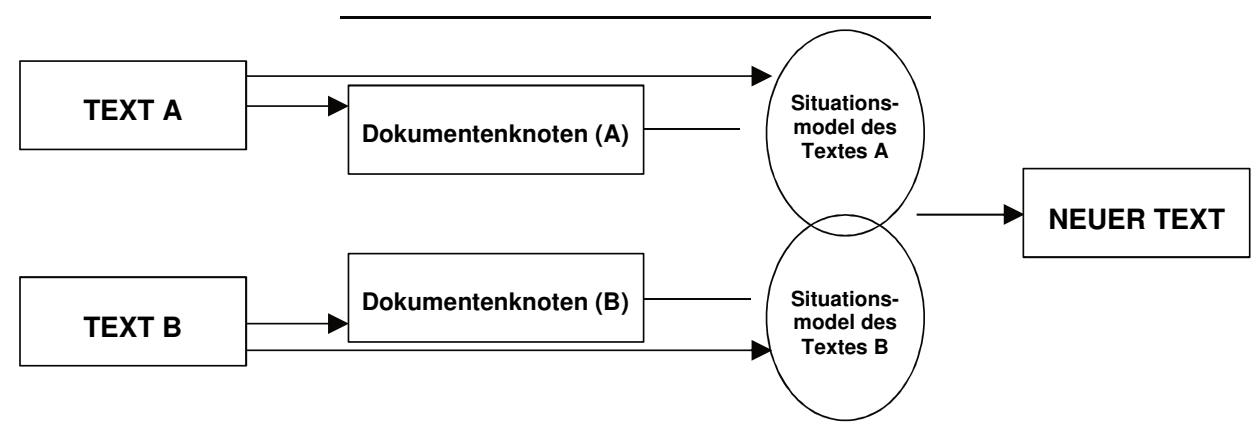

Abbildung 1. Schriftliches Erzählen ausgehend vom Gelesenen nach Britt \& Rouet (2012: 285) 
prozesses. Ein neu verfasster (Schüler)text kann andere Texte oder Elemente daraus adoptieren und mit ihnen, mit Formen und Mustern, in denen Inhalte und Themen entfaltet, und Bedeutungsstrukturen gestaltet werden, korrespondieren. „Ein Text ist immer ein Text zwischen Texten“ (Dehn 1999: 36), "Mosaik von Zitaten“ (Kristeva 1972: 248) und er ist in ein „Textuniversum" (Adamzik 2004: 244), einen globalen Diskurs eingebunden (Jung 2000). Schematisch lässt sich das in Anlehnung an das multi-dokumentmodell von Britt and Rouet (2012: 285) wie folgt darstellen (s. Abbildung 1).

Ausgehend von diesen Überlegungen sowie unter Berücksichtigung der Vorstellungen von Vygotsky (1978) zur Zone der nächsten Entwicklung und somit der Zukunftsorientierung jedes Lernprozesses (Bruner 1985) ließe sich die unterrichtliche Förderung der Erzählkompetenz anders gestalten. Im Fall des Erzählens verfügen die Schülerinnen und Schüler über das pränarrative Vermögen - das rezeptiv vorhandene implizite, unbewusste Können, das nicht-diskursive Wissen, und das Vorwissen über unterschiedliche Arten und Weisen des Erzählens sowie über die literarischen Rezeptionsmuster bzw. literale Erfragungen -, das sie entweder durch Vorlesen, das eigenständige Lesen oder im Prozess der Mediensozialisation erworben haben. Der Unterricht kann an dieses Können und an das Vorwissen anknüpfen, es konkretisieren, bewusstmachen, erweitern, es in ein explizites, prozedurales Wissen überführen und schließlich über die traditionelle Prototypenvermittlung hinausgehen. Dies wird die Schülerinnen und Schüler beim Verfassen ihrer Erzählungen unterstützen und Analyse der durch die literaturästhetische Geformtheit vermittelten vieldimensionalen semantischen Wirkung von Sprache als ein sinnvolles und wirksames Instrument etablieren. Dabei kommen folgende Aspekte in den Blick:

\section{Formen des Erzählens und Erzählstruktur}

Erzählen und Texte, die in der Wirklichkeit als Erzählungen gelten, kennen vielfältige Formen: beschreibend, berichtend, anekdotisch, dialogisch, szenisch, fiktional oder als Erlebnis- oder Lügengeschichten angelegt, um nur einige Varianten zu nennen. Der Erzähler kann medias in res in das Geschehen einführen oder seine Geschichte mit einer Einleitung, in der die Ereignisse erst langsam entfaltet werden, $a b$ ovo oder in ultimas res vorgehen, oder Erzählungen mit metafiktionalen Einleitungen wie Kästner (2010) versehen. Das Setting kann durch eine Landschaft, eine Szene und ein Dialog als wiederkehrende Elemente dominiert werden, wie dies beispielweise in Erzählungen von Karl May (2010) der Fall ist, oder aus einem Satz bestehen, wie bei Hohler (2008). Eine Erzählung kann einen abgerundeten Schluss haben, oder ein abruptes, ein pointiertes oder ein offenes Ende. Der Erzähler kann Spannung schrittweise aufbauen und in seinem Leser Hoffnungen 
wecken, die sich erfüllen. Er kann aber auch die zunächst aufgebauten Erwartungen enttäuschen - wie dies Wilhelm Busch (1982) in der Erzählung Eine Nachtgeschichte tut, oder ein höchst irritierendes oder sehr offensichtliches Netz von Anspielungen, Parallelen und Oppositionen anlegen, wie Rowling in Harry Potter, Moers 2007 in Die Stadt der träumenden Bücher oder Funke in der Trilogie Tintenwelt (2013) oder Reckless (2010).

\section{Der Anlass}

Es kann richtig sein, dass man von etwas Besonderem, das vorgefallen ist, erzählen kann, eine Bedingung, die in erwähnten Erzähltheorien ausgewiesen wird, ein notwendiger Anlass dafür, ist dies jedoch nicht. Denn alles Erzählte bekommt seinen Stellenwert erst dadurch, dass es erzählt wird:

Dafür, dass das banalste Ereignis ein Abenteuer werde, ist es notwendig und ausreichend sich daran zu begeben, es zu erzählen. Der Mensch ist immer ein Erzähler von Geschichten. Er lebt umgeben (umschlossen) von seinen Geschichten und von den Geschichten der Anderen. Er sieht alles, was in ihm geschieht durch diese Geschichten hindurch (à travers). Und er versucht sein Leben, zu leben, wie wenn er es erzählte (Jean-Paul-Sartre 1938: 57, after/zit. nach Plügge 1962: 166).

Auch Lotman verweist darauf hin, dass das Ereignis aus einem alltäglichen Geschehen eine erzählwürdige Handlung macht (Lotman 1993). In der Wirklichkeit des Erzählens finden sich Beispiele dafür. Beispielsweise erzählt Mai in Meine ersten Minutengeschichten vom Alltag. Auch Kurzgeschichten nehmen oft als einen Anlass Ausschnitte aus dem Alltag (bspw. Hohler 2008). Erst der Akt der Narration verleiht dem Erzählten den Status von etwas Besonderem. Es geht um die erzählte Welt, um den Inhalt. Martinez und Scheffel sprechen in dieser Hinsicht von der "Was-Ebene“ des Erzählens (Martinez \& Scheffel 2009: 109). Die erzählerische Gestaltung eines Ereignisses, der Stil, Figuren und Sujetlinien, der Aufbau und der Erzähler konstituieren wiederum die „Wie-Ebene“ des Erzählens (Martinez \& Scheffel 2009: 109; Mair 2016).

Die Fähigkeit, Figuren zu charakterisieren, ist eine der anzustrebenden Kompetenzen (Eigencharakteristik und Fremdcharakteristik). Demnach ist es eine Aufgabe des Unterrichts, bei Schülerinnen und Schülern das Verständnis für das Entwickeln und für die Ausgestaltung der Figuren zu schaffen. Dies kann nicht mit Auflistungen von Adjektiven erreicht werden, sondern "durch bestimmte Szenen, die zu dieser Figur gehören und die sie auf einzigartige Weise porträtieren" (Ortheil \& Siblewski 2008: 67). Auch ist die Genrespezifik zu berücksichtigen. Figuren in Kurzgeschichten werden anders konzipiert als in Comics, in Kriminalgeschichten, in Abenteuerromanen oder in Horrorerzählungen. 
Zeit

Wie werden die erzählte, Sprech-, die Ereignis- und die Betrachtzeit sowie die Erzählzeit organisiert? Die Antworten auf diese Fragen bedingen die Wahl der Tempusformen für die Demonstration dieser Ebenen, denn „das Erzählen funktionalisiert die Zeitformen für eigene Zwecke und gestaltet Zeiterfahrung eigenständig" (Feilke 2013: 10). Beispielsweise, Lightman (1994) lässt seine Protagonistin jünger werden und erzählt aus radikal subjektiven Perspektive durchgehend in Präsens. Ähnlich ist es bei Drvenkar (2008) in touch the flame. Bei Maron (1988) in Überläuferin erinnert sich Rosalind Polkowski an ihre Gedanken noch im Mutterleib und an ihre eigene Geburt in Präsens und in Präteritum.

Interessant in dieser Hinsicht ist, dass bereits jüngere Kinder in ihren frühen fiktionalen Erzählungen das Präteritum verwenden und in das szenische Präsens für die Dynamisierung der Narration oder in das Perfekt für die Gestaltung der Dialogizität bzw. der Relevanz der Ereignisse für die Gegenwart wechseln. Dabei ist der Einfluss unterrichtlicher Implikationen auszuschließen (Quasthoff 2002: 119). Ohlhus (2005) kommt zum Schluss, dass Kinder bereits früh eine Aufmerksamkeit für das Präteritum als Mittel zur Markierung der Versetzung in einem fiktionalen Erzählraum haben. Lehrwerke für Deutschunterricht betonen die Verwendung des Präteritums dezidiert und weisen explizit auf die Einhaltung der gewählten Zeit hin, was allerdings dazu führt, das Lernende in einen Konflikt geraten, zwischen ihren literarischen Erfahrungen und den aufgestellten Regeln eines prototypischen Musters.

\section{Spannung}

Spannung muss man sich nicht immer mit eiskaltem Schauern und Gänsehaut vorstellen (Ruf 2000). Es kann auch die Spannung auf den Fortgang der Geschichte sein, auf die nächste Episode. Caroll (2015) schickt Alice von Station zu Station und erzeugt dadurch Spannung. In Die Insel der Tausend Gefahren von Packard (1996) wird die Spannung durch die hypertextuelle Struktur erzeugt, wie auch in der virtuellen Hyperfiction Zeit für die Bombe von Berkenheger (1997).

Spannung kann auch dadurch aufgebaut werden, dass nur ein bruchstückhaftes Bild der Szene geliefert wird, die selbst wiederum ein Bruchstück auf die ganze Geschichte vorausweist. Wie könnte es dazu kommen? (Herndorf 2010 in tschik). Spannung umfasst subtile und vielfältige Gestaltungsmöglichkeiten durch Verweise, An- und Vorausdeutungen, Allusionen, Aussparungen, Unbestimmtheitsstellen, Rückverweise, Fragen an den Leser, Hoffnungen, die man ihm macht, Einschübe, Unterbrechungen, Irre- 
führungen, parallele und gegensätzliche Elemente, die es in Erzählungen der Wirklichkeit zu erkennen und für eigene Textproduktion zu nutzen gilt. Sie ist eine kontextbedingte Grammatik und Semantik des Textes und folgt keiner Regel. Das Konzept der Spannung ist kein universelles. Die Vorstellungen der Leser zu unterschiedlichen Zeiten und in unterschiedlichen Kulturen von dem, was spannend ist, differieren und bewegen sich in einem Kontinuum mit den beiden Polen Trivialität und Spannung.

\section{Sprachliche Gestaltung}

Es geht um eine bewusste Wahrnehmung der ästhetischen Performanz der Sprache, der Wertigkeit von Wörtern, Sätzen und Aussagen im Textsystem sowie um das Erkennen der Wirkung von sprachlichen Elementen und ihrer Funktionalität zwecks einer eigenständigen produktiven Verwendung in eigenen Erzählungen: Welche lexikalischen, grammatischen und syntaktischen Einheiten werden gebraucht, wie werden sie kombiniert, welche stilistischen Mittel und vor aller in welcher Funktion werden eingesetzt, wie werden der Planbruch und die Pointe sprachlich ausgestaltet, falls diese in der Erzählung strukturell vorkommen? Exemplarisch kann das anhand des folgenden Beispiels veranschaulicht werden:

Es war Samstagmorgen, und Herr Taschenbier saß im Zimmer und wartete. Worauf wartete er? Das wusste Herr Taschenbier selber nicht genau. Warum er dann wartete? Das lässt sich schon eher erklären. Allerdings muss man dann mit dem Sonntag beginnen (Maar 1973: 7).

Die Gewöhnlichkeit der Situation wird im ersten einleitenden Satz markiert. Gleichzeitig wird hier der explizite Rückverweis bzw. die explizite Wiederaufnahme auf den Titel des Buches verwirklicht, Eine Woche voller Samstage (Maar 1973), jedoch noch nicht weiter erklärt. Parataxe unterstreicht die Lakonizität des Erzählstils von Maar und wirkt kindgerecht. Der zweite (elliptische) Hauptsatz, mit und angeschlossen, endet mit dem Verb warten, das jedoch nach einer Ergänzung im Akkusativ eingeleitet durch die Präposition auf verlangt (Kuzminykh 2016). Die Ellipse führt dazu, dass der Leser sich fragt - worauf er wartete - und wird durch diesen künstlerischen Griff des Autors in die Handlung miteinbezogen. Gleichzeitig wird Spannung erzeugt. Er liest die Frage und die Antwort darauf. Die Narration behält die elliptische Struktur bei und suggeriert eine dialogische Kommunikationssituation zwischen Interaktionspartnern, wobei dem einen von ihnen ein Wissensvorsprung inhärent ist. Warum? - in dem darauffolgenden Satz, ist eine logische Fortsetzung. Linguistisch betrachtet handelt es sich hierbei erneut um eine explizite Wiederaufnahme. Dies verstärkt die bereits ge- 
weckte Neugier, regt die Phantasie an und animiert zum Weiterlesen. Der darauffolgende Satz verdeutlicht die Erzählperspektive des allwissenden Autors, der erklärt und durch die Geschichte führt. Die dialogische Erzählweise wird dadurch nur verstärkt. Für Irritationen sorgt der letzte Satz des Zitats - "Allerdings muss man dann mit dem Sonntag beginnen" (Maar 1973: 7). Hier findet sich der Bruch mit den bereits aufgestellten Erwartungen, der auch eine lesemotivierende Funktion erfüllt. Auf der sprachlichen Ebene leistet es das konzessive Adverb allerdings, das eher in der mündlichen Kommunikation verwendet wird. Auch das intensiviert den Eindruck von einem Gespräch. Das temporale Adverb dann, als eine implizite Wiederaufnahme, schafft Verbindung zwischen der Intention des Erklärens, den Bemühungen um die Vollständigkeit und Verständlichkeit der Narration sowie akzentuiert den Wissensvorsprung des Autors - er weiß, dass die Handlung nicht an einem Sonnabend, sonders an einem Sonntag begann. Für den Leser gestaltet sich die Sachlage anderes. Er ist auf den Verlauf der Erzählung angewiesen und gleichzeitig auf den Fortgang gespannt. Auch kann die Wahl des Autors für den Gebrauch des regional verwendeten Substantivs Samstag - „besonders westdeutsch, süddeutsch, österreichisch, schweizerisch" (Duden online) - und nicht in Norddeutschland und Mitteldeutschland vorkommenden Substantivs Sonnabend thematisiert werden. Man kann das auf die Herkunft des Schriftstellers, der in Schweinfurt geboren wurde, zurückführen. Man kann aber auch hinterfragen, ob ein Wortspiel mit Sonnabend möglich und ebenso elegant und einprägsam wäre, und Sams nicht Sams, sondern Sonns oder ähnlich hieße. Der Titel lautete dann Eine Woche voller Sonnabende. Es ist ein Anlass über Sprache zu reflektieren und sich mit dialektalen Verwendungsweisen und sprachlichen Varietäten zu beschäftigen, zumal dies ein in Bildungsstandards (2012) ausgewiesener Aspekt ist. Interessant ist in diesem Satz die Verwendung des unpersönlichen Pronomens man. Als ein Indikator für auktoriale Erzählsituation schafft es Distanz. Man könnte es durch das Personalpronomen ich ersetzten und die Wirkung untersuchen. Auch lohnenswert ist die Auseinandersetzung mit dem Konzept von Origo und seiner Verschiebung (Finneman 2006). Dies wird im Wesentlichen dadurch ermöglicht, dass der Leser und der Autor in einem Vorstellungsraum mental situiert werden. Die beschriebene alltägliche Situation im ersten Satz schafft eine Anknüpfung an das Vorwissen der Leser und führt zum Herstellen einer gemeinsamen Welt, zur Verwirklichung der Tiefenfunktion des Erzählens (Ehlich 1989).

Um ein weiteres Beispiel zu geben: „schau ich zum Fenster hinaus, fließt da durch mein weiches Spiegelbild die Elbe“ (Lenz 1973: 7). Hier trifft der Leser auf ein einfaches, jedoch effektives und wirksames Verfahren. Das Verb schauen wird in der Funktion des Erinnerns gebraucht. Das Erkennen 
ermöglicht dem Leser das zeitliche Verorten. Dies ist wiederum nur unter Einbeziehung des umfangreicheren Kontextes möglich, der den Prozess der Disambiguierung in den Gang setzt. So isoliert, wie hier der Satz zitiert ist, ist auch die direkte Leseart - schauen im Sinne von visuell wahrnehmen möglich. Auch wäre zu fragen, warum schauen, wie auch die vorausgehende Passage, nicht im Präteritum gebraucht wird, wenn sich die Person an die Vergangenheit erinnert. Dies führt dazu, dass Temporagebrauch und vor allem Funktionalität in den Fokus der unterrichtlichen Aufmerksamkeit gelangen. Das szenische Präsens führt an dieser Stelle zum emotionalen Involvieren und zur Versinnbildlichung der Erinnerung. Evident wird, dass der Temporagebrauch nicht einer abstrakten Vorgabe folgt, sondern sich von den Intentionen des Erzählers, zeitliche Verhältnisse zu ordnen und nachvollziehbar zu machen, Akzente zu setzen und Kontingenz zu schaffen, herleitet.

Sprachlich und literarisch interessant ist der folgende Auszug aus Rico, Oscar und Tieferschatten von Steinhöfel (2008). Der wissbegierige Oscar fragt seinen Freund Rico nach dem Tod seines Vaters.

Wie ist er denn gestorben?

Ich gab keine Antwort. Ich habe noch nie jemandem erzählt, wie Papa gestorben ist. Das geht keinem was an. Es ist eine sehr traurige Geschichte. Ich zog die Nase hoch, guckte über den Zaun auf den Spielplatz und versuchte an etwas anderes [sic!] zu denken. Zum Beispiel, ob dort auch Schippen und Förmchen und Siebe vergraben waren, und wenn, wie viele und in welchen Farben. Bestimmt waren es hunderte. Wenn ich sie ausgrub, konnte Mama sie bei ebay versteigern zusammen mit ihren Handtaschen (Steinhöfel 2008: 37).

Rico beantwortet die Frage des Freundes nicht, weil er mit Trauer und Tränen zu kämpfen hat. Der Leser bekommt einen Einblick in die innere Welt des mutigen Jungen und zwar in Form eines inneren Monologs. Dies charakterisiert gleichzeitig den Protagonisten, der bereits auf den ersten Seiten des Romans auf seine Andersartigkeit im Bereich der kognitiven Fähigkeiten hinwies, er sei ein "tiefbegabtes Kind“ (Steinhöfel 2008:11). Diese Situation macht jedoch evident, dass Rico über seine Gefühle reflektiert und sogar über Strategien verfügt, sich von traurigen Gedanken abzulenken. Es ist hilfsbereit und sozial, denn bei dem fröhlichen Gedanken an Hunderte von Schippen und Förmchen und Sieben denkt er nicht an das Spielen, was eher ein naheliegender Gedanke für ein Kind wäre, sondern an Unterstützung seiner alleinerziehenden Mutter. Elliptische Sätze, Parataxe, Klitterungen was, Gebrauch von umgangssprachlichen Wendungen wie gucken erzeugen Authentizität der kindlichen Sprachverwendung. Die Zeitform Perfekt im Satz - „Ich habe noch nie jemandem erzählt" (Steinhöfel 
2008: 37) - verdeutlicht die Dialogizität und verbindet Vergangenheit und Gegenwart.

Und schließlich kann die Atmosphäre, die mittels Sprache kreiert wird, thematisiert werden. Süskind gelingt es in seinem Roman Das Parfüm, den Leser dazu zu bewegen, Paris im 18. Jahrhundert sowohl zu sehen als auch $\mathrm{zu}$ riechen. Er verführt ihn mittels meisterhaft ausgewählten stilistischen Mittel ins Reich der flüchtigen, olfaktorischen Empfindungen (Süskind 1994: 5). Nicht weniger ergiebig für die Thematisierung der sprachlichen Ästhetik ist der Ausschnitt mit Holz (Süskind 1994: 32). Alternativ bietet es sich an, für jüngere Schülerinnen und Schüler beispielsweise, mit dem folgenden Auszug zu arbeiten:

Das Schönste für Dietrich, den Regenschirm [K.K.] war, im prasselnden Regen draußen die Familie Wendland gegen den Regen zu schützen. Dietrich liebte es, wenn der Regen auf ihn prasselte und in Sturzbächen von Schirm floss. Er mochte es aber auch, wenn sich ganz feiner Nieselregen fast behutsam auf ihm absetzte, wenn dann einzelne Tropfen sich langsam bildeten und vorn, hinten oder an den Seiten hinabliefen. An manchen Tagen stürmte es, und der Regen schien fast waagerecht von der Seite zu kommen [...] (Gieseking 2013: 33).

Die Wirkung entsteht hier dadurch, dass der Autor die vielfaltigen Arten des Regens in einer gelungenen Kombination von (onomatopoetischen) Verben, Adjektiven und Substantiven darstellt. „Dietrich liebte“ es - die Wahl des Verbs lieben verstärkt die Aussage, die im weiteren Verlauf durch „Er mochte es“ explizit wiederaufgenommen wird: Dadurch vermeidet der Autor Wiederholungen. Partikel "aber" betont die neutrale Semantik des Verbs mögen. Interessant ist die Darstellung von unterschiedlichen Arten des Regens. Es kommen nicht nur die Bezeichnungen der Regenarten in den Blick, sondern die Einstellung des Regenschirms dazu und die beschreibende Erzählweise. Man kann über Synonymie reflektieren.

\section{Erzählperspektive}

Die Wahl der Erzählperspektive und die Konsequenzen für die Narration sind ebenfalls in den Texten der Wirklichkeit zu erkunden. Der IchErzähler, wie beispielsweise in John Greens (2012) Das Schicksal ist ein mieser Verräter wirkt authentisch, direkt, nah und unmittelbar. Bei Green ist das die Ich-Erzählerin Hazel Grace, die ihre Geschichte erzählt, ihre eigenen Gefühle und Gedanken, Wahrnehmungen und ihre Sicht der Ereignisse erinnernd oder erlebend darlegt, doch kann sie nicht einen Einblick in das Innenleben der anderen Figuren gewähren. Sie kann nur aufgrund ihrer ausgeprägten Empathie und ihrer scharfsinnigen Beobachtungen Vermutungen über die 
innere Welt der anderen aufstellen. Dies leistet der allwissende auktoriale Erzähler. Er überblickt aus der Distanz von außen Motive aller Handelnden, dafür büßt er an der Authentizität ein, wie beispielsweise bei Maar (1973) in Eine Woche voller Samstage oder bei Kästner (2010) in Emil und Detektive. Kreitz (2012) lässt in dem gleichnamigen Comic die Figuren selbst sprechen. Dies kann durch die Medienspezifik erklärt werden, wenngleich sie auch in der Einleitung den Autor aus der auktorialer Perspektive zum Wort kommen lässt und im Fortlauf der Geschichte auf seine Kommentare nicht vollständig verzichtet (Analyse bei Kuzminykh 2014; 2015).

Das Geschehen muss weder aus der Sicht einer Person erzählt werden noch braucht es einen kommentierenden Erzähler. Fakten und Vorgange werden sachlich - wie beispielsweise in Fabeln oder Erzählungen der Aufklärungszeit oder in Max und Moritz (Busch 1984) - wiedergegeben (Wangerin 2011) oder wie dies Gieseking (2013) in der Erzählung Dietrich, der Regenschirm zu Beginn der Narration nahezu ohne emotionale Färbung dargelegt:

Dietrich, der Regenschirm, steckte in einer Regenschirmhülle und hing bei der Familie Wendland in Hausflur an der Garderobe. Meistens jedenfalls. Es sei denn, es regnete. Dann durfte er raus. Dann zeigte er sich draußen der Welt, weit aufgespannt, in seiner ganzen Pracht. Nach dem Regenschauer stand er stolz, weiter ausgespannt und ausgebreitet, im Hausflur, zum Trocknen. Hin und wieder auch im Bad, in der Badewanne (Gieseking 2013: 33).

Man kann in den Erzählungen nach solchen Momenten suchen, in denen sich Protagonisten an etwas erinnern, von ihren Gefühlen erzählen, ihre Zweifel zum Ausdruck bringen und fragen, wie das umgesetzt wird. Welche Wirkung stellt sich bei unterschiedlichen Perspektiven ein? Man kann versuchen, Perspektiven zu ändern, beispielsweise im letzten Zitat werden dem Regenschirm Wahrnehmungsweisen und Einstellungen zugewiesenen, die nicht unbedingt mit den Wahrnehmungen der Leser korrelieren. In dem weiteren Verlauf des Textes wünscht sich Dietrich ein Jahr Regen - ein Wunsch, den im nördlichen Europa die Wenigsten hegen, in Ländern, in denen Dürre und Hitze herrschen, mag das anders aussehen, und aus der Sicht des Regenschirms, erscheint dieser Gedanke nicht abwegig. Hier erreicht der Autor einen Verfremdungseffekt, die Alterität.

Als ein Mittel der Nähe oder Distanz kann die Redewiedergabe fungieren. Sehr überzeugend verwirklicht das Canetti (2012: 7) in Blendung, in dem, im ersten Kapitel die Begegnung zwischen Franz Menzinger und Professor Peter Kien zunächst dialogisch und anschließend in Form eines Erzählberichts zu lesen ist. Dies lädt zu eigenen Experimenten ein. Einmal darauf aufmerksam geworden, werden die Schülerinnen und Schüler auch selbst weitere Möglichkeiten zur Gestaltung ihrer Erzählungen entdecken. 


\section{ERZÄHLEN IM INTEGRATIVEN DEUTSCHUNTERRICHT: EXEMPLARISCHE KONZENTRATION}

In der exemplarischeren Konzentration und im reflektierten Umgang mit fremden und eigenen Texten liegen Chancen, das Schreiben von Erzählungen nicht nur als Lerngegenstand zu sehen, sondern es auch als ein Medium des Lernens in den Bereichen Lesen, Reflexion über Sprache und Schreiben zu nutzen (Pohl \& Steinhoff 2010; Zabka 2005; Feilke 2013).

Konkret ließe sich das umsetzen, wenn Schülerinnen und Schüler gebeten werden, Texte, die sie für Erzählungen halten, in den Unterricht zu bringen. Dabei ist stets zu berücksichtigen, dass der Erzähldiskurs der offenste ist, der am meisten zulässt und am wenigsten einheitlich zu bestimmen ist (Karg 2009). Die Lehrperson verschafft sich dadurch eine Vorstellung, von dem, was den Schülerinnen und Schülern an Prätexten - hier nicht im Sinne von Schnittstellen zwischen Denken und Niederschrift unmittelbar im Prozess des Formulierens gemeint (Pohl \& Steinhoff 2010: 13), sondern im Sinne des Erzählvermögens - zur Verfügung steht, und welche Vorstellung sie vom Erzählen haben. Mit Sicherheit werden sich bei ihnen Assoziationen einstellen und sie werden sich an Texte aus ihrer eigenen Leseerfahrung erinnern. Es wird dadurch ein gemeinsamer Wissensbestand gesichert und eventuell sogar Lesemotivation und -neugier erzeugt. Ferner ist eine Anbindung an Buchpräsentationen, die ein Bestandteil des Deutschunterrichts am Ende der Primarstufe und zu Beginn der Sekundarstufe I sind (Bildungsstandards im Fach Deutsch 2004) möglich. Auch lassen sich (Vor)lesewettbewerbe, Lesetagebücher oder Leseportfolios, Lesenächte u. Ä. sinnvoll einbinden.

Die Lehrperson sucht auch nach Erzählungen, die möglichst diverse Erzählstrukturen aufweisen:

- Erzählungen, die reihend oder episodenhaft aufgebaut sind, eine (a)chronologischen Darstellung der Wirklichkeit oder eines (un)spektakulären Alltags enthalten,

- Erzählungen, in denen die Erzählzeit und die erzählte Zeit übereinstimmen, und in denen diese nicht identisch sind,

- Erzählungen mit Einleitungen, die mit den gezielt eingesetzten Mitteln bewirken, dass der Leser jeweils eine bestimmte Situation imaginiert,

- Erzählungen mit Einleitungen, die das Geschehen langsam entfalten und den Leser lenken,

- Erzählungen mit Einstiegen medias in res, ab ovo und in ultimas res,

- Erzählungen mit metafiktionalen Einleitungen,

- Erzähltexte, die ihre erzählte Welt nicht in einem harmonischen Schluss auflösen, sondern den Leser mit Irritationen herausfordern, 
- Erzählungen, die auf einer vielfachen Verschränkung von Binnen- und Rahmenerzählung beruhen und ein hohes Maß an Kombinationstätigkeit beim Lesen erfordern,

- Erzählungen, die bestimmte Themen und Motive akzentuieren,

- Texte mit dem Handlungsaufbau, bei dem die Spannung kontinuierlich ansteigt, und solche die darauf verzichten.

Dies ist notwendig, um den Lernprozess kontrollieren und zielgerichtet steuern zu können. Andernfalls kommt es zu einer Situation, vor der bereits Mayer 2004 gewarnt hat: The formula hands on activity is a formula for educational disaster (Mayer 2004: 17). Die Erkundung soll eine bewusstgemachte Rezeption, ein input, ein teaching-learning-cycle (Martin 2009: 16) sein. Dabei kämen die bereits angesprochenen Aspekte der verstehenden Lektüre in den Blick. Die Lehrperson muss eventuell eine kompensatorische Arbeit leisten, besonders, wenn Texte sprachliche, historische und kulturelle Distanz aufweisen, und da das Lesen als Vorstufe für das Verfassen von eigenen Texten aufzufassen ist, wären in den authentischen Erzählungen Elemente zu eruieren, die in der eigenen Textproduktion produktiv genutzt werden könnten. Als Gestaltungskomponenten des literalen sprachlichen Duktus können folgende auftreten:

- Mittel, mit denen bspw. Spannung aufgebaut wird, und Kontexte, wie dies geschieht - was wirkt spannend in den Erzählungen?

- Setting und seine Ausgestaltungsmöglichkeiten: Gestaltungsmittel für den Einstieg, für den Schluss, für den potenziellen Planbruch und für die Pointe,

- Baustruktur der Erzählung und Erzählperspektiven,

- Formulierungen zur „emotionalen Anreicherung“ (Augst 2010: 92), zum narrativen Beschreiben und Charakterisierung der Figuren,

- Strukturen und Mittel, die Kohäsion und Kohärenz erzeugen, die Lexikogrammatik. Dies ermöglicht eine additive Aneinanderreihung - die listenförmige "Stereotypie des Satzes“ (Feilke 1995: 82) - möglichst gering zu halten und die Schülerinnen und Schüler dazu zu befähigen, einen konsistenteren Text zu verfassen,

- Gebrauch der direkten Rede als eine Form des Mis-es-Scene, der Nähe und Distanz und als ein Mittel zur Involvierung des Lesers,

- Gebrauch von Tempusformen, der nicht einfach einer Regel folgt, sondern sich als eine Antwort auf eine Frage ergibt, an welcher Stelle des Textes, auf welche Einzelzeit zu verweisen ist, um das Konstrukt der erzählten Welt aufzubauen und Gleichzeitiges, Ungleichzeitiges, Vorzeitiges und Nachgeholtes zu signalisieren und sinngemäß miteinander in Beziehung zu setzen. Aspekte der Raffung und Zeitdehnung 
und sprachliche Mittel, mit denen diese erreicht werden, sind zu fokussieren,

- bei fantastischen Erzählungen neben den Mitteln zur Gestaltung des Planbruchs, die Gestaltung der Rückkehr zur Normalität. Man kann an dieser Stelle in Ansätzen das Modell von Campbell (2008) The hero with a thousand faces thematisieren. Es wäre ein Propädeutikum für die in der siebten und in der achten Klassen beginnende filmanalytische Arbeit.

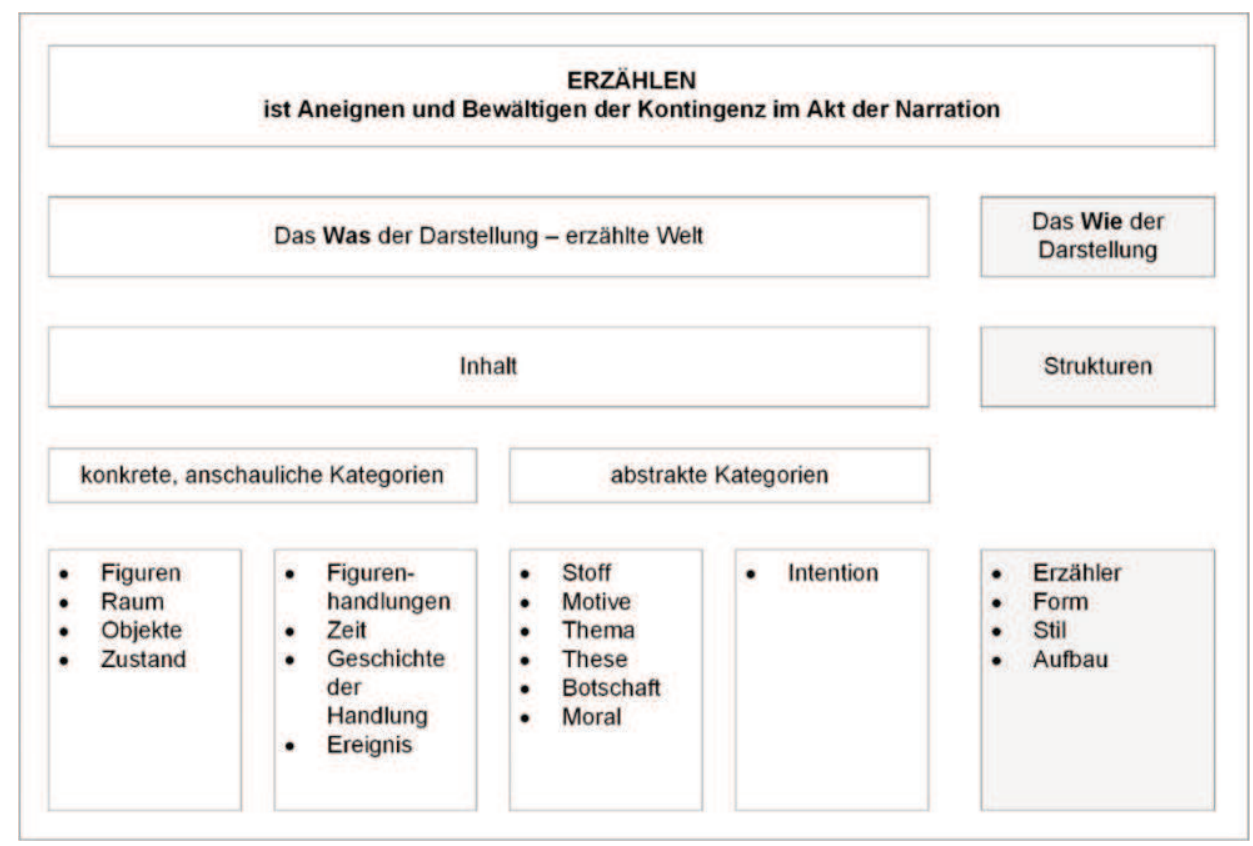

Abbildung 2. Anregungen zur Erkundung des Erzähldiskurses (eigene Darstellung)

Sie könnten in den Gruppenarbeiten zunächst erkundet und aufgeschrieben und in einem weiteren Schritt für alle verfügbar gemacht werden. In werkstattähnlichen Lernarrangements können gemeinsames Auswählen von erzählerischen Vorlagen, der erzählten Zeit und der epischen Welt, das Planen und die Strukturierungen des zu Erzählenden und sogar das Formulieren und besonders das Überarbeiten von Gestaltungsvarianten produktiv verwirklicht werden. Abbildungen 2 und 3 enthalten mögliche Anregungen für die Erkundung und eigene Schreibproduktion, die an die jeweilige Lerngruppe angepasst werden können. 


\begin{tabular}{|c|c|c|c|}
\hline \multicolumn{4}{|c|}{$\begin{array}{l}\text { ERZÄHLEN } \\
\text { ist Aneignen und Bewältigen der Kontingenz im Akt der Narration }\end{array}$} \\
\hline $\begin{array}{l}\text { Thema, Inhalt und Gegenstand } \\
\text { - Was soll erzählend bearbeitet } \\
\text { werden? } \\
\text { - Wird eigene Erfahrungswelt } \\
\text { bearbeitet, aufgearbeitet oder } \\
\text { erzählt? } \\
\text { - Wird etwas den anderen } \\
\text { mitgeteilt, was man fur wichtg } \\
\text { erachtet? } \\
\text { - Werden phantastische Welten } \\
\text { kreiert? } \\
\text { - Soll das Prinzip der } \\
\text { (steigernden) Fiktionalität bzw. } \\
\text { Spannung zum Einsatz } \\
\text { kommen? } \\
\text { - Werden lustige, satirische oder } \\
\text { gruselige Geschichten } \\
\text { verfasst? } \\
\text { - Werden Lügengeschichten } \\
\text { oder Kriminalgeschichten } \\
\text { entstehen? } \\
\text { - Wird etwas problematisiert? } \\
\text { Werden bestimmte } \\
\text { Geschehnisse akzentuiert? } \\
\text { Wie? }\end{array}$ & $\begin{array}{l}\text { Struktur und Konstitution der } \\
\text { erzählten Welt } \\
\text { - Welche Uberlegungen } \\
\text { bedingen die Wahl der } \\
\text { erzahlten Zeit und der } \\
\text { Sprechzeit, der Ereigniszeit } \\
\text { und die Betrachtzeit? } \\
\text { - Wann ereigneten sich Fakten, } \\
\text { von denen erzahlt wird? } \\
\text { - Wie sollen die einzelnen } \\
\text { Zeitebenen in Beziehung } \\
\text { gesetzt werden? } \\
\text { - An welcher Stelle des Textes } \\
\text { ist auf weiche Zeit mit welchen } \\
\text { Mitteln zu verweisen? } \\
\text { Wie können chronologische } \\
\text { und kausallogische } \\
\text { Beziehungen hergestellt } \\
\text { werden? } \\
\text { Welche grammatischen und } \\
\text { lexikalischen Strukturen stehen } \\
\text { zur Verfügung, um die } \\
\text { Zeitverhältnisse, Spannung } \\
\text { und Logik in der Erzählung zu } \\
\text { verdeutlichen? }\end{array}$ & $\begin{array}{l}\text { - Mit welchen grammatischen, } \\
\text { lexikalischen und syntaktischen } \\
\text { Mittel wird ein kohărenter und } \\
\text { ein kohäsiver Text geschaffen? } \\
\text { - Wie werden explizite und } \\
\text { implizite Wiederaufnahmen } \\
\text { verwirklicht? } \\
\text { - Wie werden die Pointe und der } \\
\text { Planbruch ausgestaltet? } \\
\text { - Wie wird Spannung aufgebaut? } \\
\text { - Welche Andeutungen und } \\
\text { Verweise mussen erfolgen, } \\
\text { damit der Text spannend wirkt, } \\
\text { und welche Informationen } \\
\text { können ausgespart werden, } \\
\text { ohne dass Irritationen } \\
\text { entstehen? } \\
\text { - Wie wird der Leser durch den } \\
\text { Text gefuhrt? } \\
\text { Welche sprachlich-literarischen } \\
\text { Mittel können verwendet } \\
\text { werden? } \\
\text { Wie wird Ästhetizität } \\
\text { verwirklicht? }\end{array}$ & $\begin{array}{l}\text { - Wie werden Prinzipien der } \\
\text { Erzählweise und eines } \\
\text { konsistenten Aufbaus sowie die } \\
\text { Komplexitat des Erzählens } \\
\text { verwirklicht? } \\
\text { - Wie kann ein Einstieg in die } \\
\text { Erzählung erfolgen - medias in } \\
\text { res, ab ovo oder in ultimas res? } \\
\text { - Wie werden Figuren und Orte } \\
\text { konstituiert? } \\
\text { - Wie werden die } \\
\text { Einzelgeschehnisse arrangiert? } \\
\text { - Wie wird die Geschichte } \\
\text { abgeschlossen? } \\
\text { - Orientierung am Leser: } \\
\text { Welches Wissen und welche } \\
\text { Erfahrung kann beim Leser } \\
\text { antizipiert werden - Prinzip } \\
\text { aptum? } \\
\text { Können Erzähltraditionen und } \\
\text { Muster anderer Epochen und } \\
\text { Kulturen einbezogen werden? }\end{array}$ \\
\hline
\end{tabular}

Abbildung 3. Anregungen zur Erkundung des Erzähldiskurses (eigene Darstellung)

Eine solche Herangehensweise gründet auf dem Konzept der Transformationsparadigmata, dem Ansatz reading-to-write, der Modellierung des Schreibens und des Lesens als kognitive Konstruktionsprozesse (Prozessorientierung unter einer Berücksichtigung des Produktes und der Wirklichkeit der schriftsprachlichen Kommunikation, hier des Erzähldiskurses) und der sozialen Kohärenz. Sie evoziert integrative sprachlich-literarische Lernprozesse vor dem Hintergrund der Arbeit mit diversesten Facetten der Erzählliteratur und befähigt Schülerinnen und Schüler zum sinnstiftenden Erzählen und zur Entwicklung des individuellen Erzähltons.

\section{LITERATURVERZEICHNIS}

Adamzik, K. (2004). Sprache: Wege zum Verstehen. Tübingen: Francke.

Augst, G. (2010). Zur Ontogenese der Erzählungskompetenz in der Primar- und Sekundarstufe. In: T. Pohl / T. Steinhoff (Hrsg.), Textformen als Lernformen (S. 63-96). Duisburg: Gilles \& Franke.

Augst, G. / Disselhoff, K. / Henrich, A. / Pohl, T. (2007). Text-Sorten-Kompetenz. Eine echte Longitudinalstudie zur Entwicklung der Textkompetenz im Grundschulalter. Frankfurt a.M.: Peter Lang. 
Becker, T. (2001). Kinder lernen erzählen. Baltmannsweiler: Schneider Hohengehren.

Bildungsstandards im Fach Deutsch für die Allgemeine Hochschulreife (2012). München: Neuwied.

Bildungsstandards im Fach Deutsch für die Primarstufe (2004). München: Neuwied.

Boueke, D. / Schülein, F. / Büscher, H. / Terhorst, E. / Wolf, D. (1995). Wie Kinder erzählen. Untersuchungen zur Erzähltheorie und zur Entwicklung narrativer Fähigkeiten. München: Fink.

Britt, M. / Rouet, J.-F. (2012). Learning with multiple documents. Component skills and their acquisitions. In: J. R. Kirby / M. J. Lawson (ed.), Enhancing the quality of learning. Dispositions, instruction, and learning process (pp. 276-314). Cambridge: CUP.

Bruner, J. (1985). Vygotsky: a historical and conceptual perspective. In: J. Wertsch (ed.), Culture, communication and cognition. Vygotskian perspectives (pp. 21-35). Cambrigde: CUP.

Campbell, J. (2008). The hero with a thousand faces. Navato: New World Library.

Dehn, M. (1999). Text und Kontext. Schreiben als kulturelle Tätigkeit in der Grundschule. Düsseldorf: Kamp.

Dittmar, N. / Thielicke, E. (1979). Der Niederschlag von Erfahrungen ausländischer Arbeiten mit dem institutionellen Kontext des Arbeitsplatzes in Erzählungen. In: H. G. Soeffener (Hrsg.), Interpretative Verfahren in den Sozial- und Textwissenschaften (S. 65-103). Stuttgart: Metzler.

Duden online (2017). www.duden.de.

Ehlich, K. (1980). Erzählen im Alltag. Frankfurt a.M.: Suhrkamp.

Ehlich, K. (1984). Zum Textbegriff. In: A. Rothkegel / B. Sandig (Hrsg.), Text - Textsorten Semantik. Linguistische Modelle und maschinelle Verfahren (S. 9-25). Hamburg: Buske.

Ehlich, K. (1989). Zur Genese von Textformen. Prolegomena zu einer pragmatischen Texttypologie. In: G. Antos / H. P. Krings (Hrsg.), Textproduktion. Ein interdisziplinärer Forschungsüberblick (S. 84-99). Tübingen: Niemeyer.

Feilke, H. (1995). Auf dem Weg zum Text. Die Entwicklung der Textkompetenz im Grundschulalter. In: G. Augst (Hrsg.), Frühes Schreiben. Studien zur Ontogenese der Literalität (S. 69-88). Essen: Die Blaue Eule.

Feilke, H. (2003). Entwicklung schriftlich-konzeptualer Fähigkeiten. In: U. Bredel (Hrsg.), Didaktik der deutschen Sprache. Ein Handbuch (S. 178-192). Paderborn: Schöningh.

Feilke, H. (2013). Erzählen gestalten - Erzählungen schreiben. Praxis Deutsch, 239, S. 4-12.

Finnemann, J. (2006). Erzählen in zwei Sprachen, Diskursanalytische Untersuchungen von Erzählungen auf Deutsch und Französisch. Münster: Waxmann.

Fix, M. (2006). Texte schreiben - Schreibprozesse im Deutschunterricht. Paderborn: Schöningh.

Fix, M. (2008). Lernen durch Schreiben. Praxis Deutsch, 210, S. 6-16.

Frederking, V. / Krommer, A. / Maiwald, K. (2012). Mediendidaktik Deutsch: Eine Einführung. Leipzig: Erich Schmidt.

Iser, W. (1976). Der Akt des Lesens. Theorie ästhetischer Wirkung. München: Beck.

Jung, M. (2000). Diskurshistorische Analyse als linguistischer Ansatz. Sprache und Literatur in Wissenschaft und Unterricht, 31, S. 20-39.

Kallmeyer, W. / Meyer-Hermann, R. (1980). Textlinguistik. In: H. P. Althaus / H. Henne / H. E. Wiegand (Hrsg.), Lexikon der Germanistischen Linguistik (S. 242-258). Tübingen: Niemeyer.

Karg, I. (2009). Diskursfähigkeit als Paradigma schulischen Schreibens. Ein Weg aus dem Dilemma zwischen Aufsatz und Schreiben. Frankfurt a.M.: Peter Lang.

Kintsch, W. (1998). Comprehension: a paradigm for cognition. Cambridge: CUP.

Knapp, W. (2001). Erzähltheorie und Erzählerwerb. Didaktik Deutsch, 10, S. 28-48.

Kristeva, J. (1972). Probleme der Textstrukturation. In: H. Blumensath (Hrsg.), Strukturalismus in der Literaturwissenschaft (S. 243-262). Köln: Rädler. 
Kuzminykh, K. (2009). Das Internet im Deutschunterricht. Ein Konzept der muttersprachlichen und der fremdsprachlichen Lese- und Schreibdidaktik. Frankfurt a.M.: Peter Lang.

Kuzminykh, K. (2014). Comics und graphic novels im multikulturellen Deutschunterricht. Glottodidactica - an international journal of applied linguistics, XLI (2), S. 75-87.

Kuzminykh, K. (2015). Emil und die Detektive. Ein Unterrichtsmodell zum Comic von I. Kreitz. http://webdoc.sub.gwdg.de/pub/mon/2015/kuzminykh.pdf. [15.03.2017]

Kuzminykh, K. (2016). Sprachreflexive Kompetenz und grammatisches Lernen mit mobilen Endgeräten im Deutschunterricht. In: M. Schuhen / M. Froitzheim (Hrsg.), Das Elektronische Schulbuch 2016. Fachdidaktische Anforderungen und Ideen treffen auf Lösungsvorschläge der Informatik (S. 165-183), Münster: LIT Verlag.

Labov, W. / Walezky, J. (1973). Erzählanalyse. Mündliche Versionen persönlicher Erfahrung. In: J. Ihwe (Hrsg.), Literaturwissenschaft und Linguistik (S. 78-126). Frankfurt a.M.: FischerÄthenäum.

Lotman, J. (1993). Die Struktur literarischer Texte. München: Fink.

Mair, M. (2016). Erzähltextanalyse. Modelle, Kategorien, Parameter. Stuttgart: Ibedem.

Maiwald, K. (2011). Erzählt wird im Präteritum. In: O. Ernst / J. Freienstein / L. Schaipp (Hrsg.), Populäre Irrtümer über Sprache (S. 59-72). Stuttgart: Reclam.

Mandler, J. / Johnson N. (1978). Erzählstruktur und Erinnerungsleistung. Eine Grammatik einfacher Geschichten. In: W. Haubrichs (Hrsg.), Erzählforschung 3. Theorien und Modelle und Methoden der Narrativik (S. 337-379). Göttingen: Vandenhoek \& Ruprecht.

Martin, J. (2009). Genre and language learning. A social semiotic perspective. Linguistics and Education, 20, s. 10-21.

Martinez, M. / Scheffel, M. (2009). Einführung in die Erzähltheorie. München: Beck.

Mayer, R. (2004). "Should there be a three strikes rule against pure discovery learning?" The case for guided methods of instruction. American Psychologist, 59, s. 14-19.

Ohlhus, S. (2005). Schreibentwicklung und mündliche Strukturierungsfähigkeiten. In: H. Feilke / R. Schmidlin (Hrsg.), Literale Textentwicklung. Untersuchungen zum Erwerb von Textkompetenz (S. 43-68). Frankfurt a.M.: Peter Lang.

Ortheil, H. / Siblewski, K. (2008). Wie Romane entstehen. München: Sammlung Luchterhand.

Plügge, H. (1962). Wohlbefinden und Missbefinden: Beiträge zu einer medizinischen Anthropologie. Tübingen: Niemeyer.

Pohl, T. / Steinhoff, T. (2010). Textformen als Lernformen. In: T. Pohl / T. Steinhoff (Hrsg.), Textformen als Lernformen (S. 5-26). Duisburg: Gilles \& Franke.

Quasthoff, U. (1980). Erzählen in Gesprächen. Linguistische Untersuchungen zu Strukturen und Funktionen am Beispiel einer Kommunikationsform des Alltags. Tübingen: Narr.

Quasthoff, U. (2002). Tempusgebrauch von Kindern zwischen Mündlichkeit und Schriftlichkeit. In: C. Peschel (Hrsg.), Grammatik und Grammatikvermittlung (S. 179-198). Frankfurt a.M.: Peter Lang.

Rank, B. (1995). Wege zur Grammatik und zum Erzählen. Baltmannsweiler: Schneider Hohengehren.

Reckwitz A. (2012). Die Erfindung der Kreativität. Zum Prozess gesellschaftlicher Ästhetisierung. Berlin: Suhrkamp.

Rehbein, J. (1980). Sequentielles Erzählen. Erzählstrukturen von Immigranten bei Sozialberatungen in England. In: K. Ehlich (Hrsg.), Erzählen im Alltag (S. 64-108). Frankfurt a.M.: Suhrkamp.

Rehbein, J. (1984). Beschreiben, Berichten und Erzählen. In: K. Ehlich (Hrsg.), Erzählen in der Schule (S. 67-124). Tübingen: Narr. 
Reusser, K. / Reusser-Weyeneth, M. (1994). Verstehen. Psychologischer Prozess und didaktische Aufgabe. Bern: Hans Huber.

Ruf, U. (2000). Spannend erzählen. Praxis Deutsch, 161, S. 46-52.

Rumelhart, D. (1975). Notes in a schema for stories. In: D. Bobrow / A. Collins (ed.), Representation and understanding. Studies in cognitive science (s. 211-236). New York: Academic Press.

Schnotz, W. (2006). Was geschieht im Kopf des Lesers? Mentale Konstruktionsprozesse beim Textverstehen aus der Sicht der Psychologie und der kognitiven Linguistik. In: H. Blühdor / E. Breindl / U. Waßner (Hrsg.), Text - Verstehen. Grammatik und darüber hinaus (S. 222-238). Berlin: de Gruyter.

Thorndyke, P. (1977). Cognitive structures in comprehension and memory of narrative discourse. Cognitive Psychology, 9, pp. 77-110.

Van Dijk, T. A. (1980a). Macrostructures. An interdisciplinary study of global structures in discourse, interaction, and cognition. Hillsdale N. J.: Erlbaum.

Van Dijk, T. A. (1980b). Story comprehension. An introduction. Poetica, 9, s. 1-21.

Van Dijk, T. A. (1980c). Textwissenschaft. Eine interdisziplinäre Einführung. Tübingen: Niemeyer.

Vygotsky, L. (1978). Mind and Society. Cambridge: CUP.

Wangerin, W. (2011). Erziehung als Programm. Von der Kinderliteratur der Philanthropen zu den 'lustigen' Warn- und Strafgeschichten. In: W. Wangerin / H. Detering (Hrsg.), Der rote Wunderschirm (S. 3-10). Frankfurt a.M.: Wallstein.

Wenzel, P. (2004). Einführung in die Erzähltextanalyse. Trier: Wissenschaftlicher Verlag.

Wolf, D. (2000). Modellbildung im Forschungsbereich sprachliche Sozialisation. Frankfurt a.M.: Peter Lang.

Zabka, T. (2005). Vom Nutzen des literarischen Erzählens für die sprachliche Sozialisation. Der Deutschunterricht, 2, S. 40-48.

\section{LITERARISCHE WERKE}

Berkenheger, S. (1997). Zeit für die Bombe. http:/ / www.wargla.de/94Dollar.html [15.03.2017].

Busch, W. (1982). Eine Nachtgeschichte. In: W. Busch (Hrsg.), Und die Moral von der Geschicht (S. 10). München: Bertelsmann Verlag.

Busch, W. (1984). Max und Moritz. In: W. Busch (Hrsg.), Das neue Wilhelm Busch Album in Farbe (S. 7-26). Stuttgart: Unipart-Verlag.

Canetti, E. (2012). Blendung. Frankfurt a.M.: Fischer.

Caroll, L. (2015). Alice in Wunderland. Hildesheim: Gerstenberg Verlag.

Drvenkar, Z. (2001). Touch the flame. Hamburg: Carlsen.

Funke, C. (2010). Reckless. Hamburg: Dressler.

Funke, C. (2013). Tintenwelt. Hamburg: Dressler.

Gieseking, B. (2013). Das große Buch der Wünsche. Oldenburg: Lappan.

Green, J. (2012). Das Schicksal ist ein mieser Verräter. München: Carl Hanser.

Herndorf, W. (2010). Tschik. Berlin: Rowohlt.

Hohler, F. (2008). Das Ende eines ganz normalen Tages. München: Luchterhand.

Kästner, E. (2010). Emil und die Detektive. Hamburg: Dressler.

Kreitz, I. (2012). Emil und die Detektive. Ein Comic von Isabel Kreitz. Hamburg: Dressler.

Lenz, S. (1973). Die Deutschstunde. München: Deutscher Taschenbuch Verlag.

Lightman, A. (1994). Einstein's dream. A Novel. New York: Warner Books.

Maar, P. (1973). Eine Woche voller Samstage. München: Deutscher Taschenbuch Verlag. 
Mai, M. (2010). Meine ersten Minutengeschichten: Gute Nacht. Ravensburg: Ravensburger Buchverlag.

Maron, M. (1988). Die Überläuferin. Frankfurt a.M.: Fischer.

May, K. (2014). Winnetous größte Abenteuer. Hamburg: Arena.

Moers, W. (2007). Die Stadt der träumenden Bücher: Ein Roman aus Zamonien. München: Pieper.

Packard, E. (1996). Die Insel der Tausend Gefahren. Ravensburg: Ravensburger Buchverlag.

Rowling, J. (2005). Harry Potter und der Stein der Weisen. Hamburg: Dressler.

Steinhöfel, A. (2008). Rico, Oscar und Tieferschatten. Hamburg: Carlsen.

Süskind, P. (1994). Das Parfüm. Die Geschichte eines Mörders. Zürich: Diogenes.

Received: 15.03 .2017 ; revised: 21.06 .217 
